\title{
Energy of vacancy formation in the continuum matter model
}

\author{
A.G. Khrapak ${ }^{1}$ and S.A. Khrapak ${ }^{1,2}$ \\ ${ }^{1}$ Joint Institute for High Temperatures, Russian Academy of Sciences, 125412 Moscow, Russia \\ E-mail: khrapak@mail.ru \\ ${ }^{2}$ Max-Planck-Institut für Extraterrestrische Physik, Garching, Germany
}

Received September 24, 2012

\begin{abstract}
The quantum energy spectrum of the oscillating spherical void in solids is calculated within the continuum matter model. It is suggested that the ground state of the oscillating void corresponds to the vacancy in real crystals. The dependence of the vacancy formation energy on the shear modulus, density, pressure and surface tension is determined. The obtained results are used to estimate properties of vacancies in solid Ar. A possibility to use the obtained results to estimate the properties of vacancies in liquid melts is discussed.
\end{abstract}

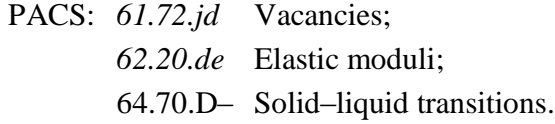

Keywords: vacancy, shear modulus, melting.
Many properties of crystals near melting are determined by presence of vacancies [1]. First of all, this concerns the processes of the self-diffusion, the diffusion of impurities, the ion mobility, etc. Vacancies also influence the specific heat and volume thermal expansion of crystals. A number of attempts to construct the vacancy model of melting are known [2-4]. Molecular dynamics studies of melting reveal that an ideal crystal melts at temperatures notably higher than a crystal that contains vacancies $[5,6]$. The energy of the vacancy formation $E_{v}$ is the most important characteristic of the vacancies. Experimental values of $E_{v}$ near the triple point are known for many substances, including series of metals [7] and rare gas solids [8]. Attempts of theoretical determination of $E_{v}$ are very cumbersome, which makes difficult their extrapolation to the limit of extremely high pressures and temperatures. In the present paper a simple model is suggested, which gives a possibility to obtain an analytical dependence of $E_{v}$ on the crystal density, external pressure and shear modulus.

Within the model of an isotropic matter, a spherical void formation results in the local change of the crystal potential energy due to the deformation and work against the surface and volume forces:

$$
\Delta U=U_{d}+U_{s}+U_{v}=U_{d}+4 \pi \sigma R^{2}+(4 \pi / 3) p R^{3},
$$

where $\sigma$ is the surface tension, $p$ is the external pressure, and $R$ is the void radius. In equilibrium, the energy of deformation is given by [9]

$$
U_{d}=\int\left[\frac{\lambda}{2} u_{i i}^{2}+\mu u_{i k}^{2}\right] d^{3} r,
$$

where $\lambda$ and $\mu$ are the Lamé coefficients, and $u_{i k}$ is the strain tensor, which (for small deformations) reads

$$
u_{i k} \cong \frac{1}{2}\left(\frac{d u_{i}}{d x_{k}}+\frac{d u_{k}}{d x_{i}}\right) .
$$

In the case of a spherical void, the vector of deformation $\mathbf{u}$ depends only on spherical coordinate $\mathbf{r}$

$$
\mathbf{u}=u(r) \mathbf{n}_{r}
$$

and nonzero components of the deformation tensor are

$$
u_{r r}=\frac{d u}{d r}, \quad u_{\theta \theta}=u_{\varphi \varphi}=\frac{u}{r} .
$$

The quadratic forms included in Eq. (2) are given by

$$
\begin{aligned}
& u_{i i}^{2}=\left(u_{r r}+u_{\theta \theta}+u_{\varphi \varphi}\right)^{2}=\left(\frac{d u}{d r}+2 \frac{u}{r}\right)^{2}, \\
& u_{i k}^{2}=u_{r r}^{2}+u_{\theta \theta}^{2}+u_{\varphi \varphi}^{2}=\left(\frac{d u}{d r}\right)^{2}+2\left(\frac{u}{r}\right)^{2} .
\end{aligned}
$$


The equation of equilibrium of an isotropic incompressible solid is [9]

$$
(\lambda+\mu) \operatorname{grad} \operatorname{div} \mathbf{u}+\mu \Delta \mathbf{u}=0 .
$$

In the spherically symmetric case $\operatorname{rot} \mathbf{u}=0$ and Eq. (7) gets a form

$$
\operatorname{grad} \operatorname{div} \mathbf{u}=0 .
$$

From this it follows that $u$ satisfies equation

$$
\operatorname{div} \mathbf{u}=\text { const },
$$

solution of which finite outside the void is

$$
u=a / r^{2} \text {. }
$$

The constant $a$ is determined from the boundary condition

$$
u(R)=a / R^{2}=R, \quad a=R^{3} .
$$

Thus

$$
u(r)=\frac{R^{3}}{r^{2}}, \quad u_{i i}^{2}=0, \quad u_{i k}^{2}=6 \frac{R^{6}}{r^{6}} .
$$

Now Eq. (2) gets a form

$$
U_{d}=8 \pi \mu R^{3}
$$

Taking into account the kinetic energy of the radial motion, the change of the full energy of the deformed isotropic matter is given by

$$
\begin{aligned}
& \Delta E=\int \frac{\rho \dot{\mathbf{u}}^{2}}{2} d^{3} r+4 \pi \sigma R^{2}+\frac{4 \pi}{3} p R^{3}+8 \pi \mu R^{3}= \\
& =6 \pi \rho R^{3} \dot{R}^{2}+4 \pi \sigma R^{2}+\left(8 \pi \mu+\frac{4 \pi}{3} p\right) R^{3},
\end{aligned}
$$

where $\rho$ is the matter density and $\dot{u}=3 R^{2} \dot{R} / r^{2}$. In the case of $\Delta E \neq 0$ this equation describes an oscillatory motion of the void wall under the action of the elastic forces, surface tension and pressure. The energy of any oscillation is quantized. The dispersion law of these oscillations can be determined from the Bohr-Sommerfeld quantization conditions

$$
\oint P_{R} d R=2 \pi \hbar\left(n+\frac{3}{4}\right), n=0,1, \ldots
$$

where $P_{R}=4 \pi \rho R^{3} \dot{R}$ is the generalized momentum of the surrounding matter. Substitution of $\dot{R}$ from Eq. (14) into Eq. (15) gives an integral equation for determination of the void creation energy $\Delta E_{n}$

$$
\begin{aligned}
& \int_{0}^{R_{n}} R^{3 / 2}\left[\Delta E_{n}-4 \pi \sigma R^{2}-\frac{4 \pi}{3}(6 \mu+p) R^{3}\right]^{1 / 2} d R= \\
& =\left(\frac{3 \pi}{8 \rho}\right)^{1 / 2} \hbar\left(n+\frac{3}{4}\right),
\end{aligned}
$$

where the maximum size of the void $R_{n}$ is determined by

$$
\Delta E_{n}-4 \pi \sigma R_{n}^{2}-\frac{4 \pi}{3}(6 \mu+p) R_{n}^{3}=0
$$

In two limiting cases, solution of Eqs. (16) and (17) can be obtained analytically. When $(6 \mu+p) R_{n} / 3 \sigma \gg 1$ the surface force can be neglected and we get

$$
\Delta E_{n} \cong \frac{[8 \pi \mu+(4 \pi / 3) p]^{5 / 8}}{I^{3 / 4}}\left(\frac{3 \pi}{8 \rho}\right)^{3 / 8} \hbar^{3 / 4}\left(n+\frac{3}{4}\right)^{3 / 4},
$$

$R_{n}^{3} \cong \frac{\Delta E_{n}}{8 \pi \mu+(4 \pi / 3) p}$,

where

$$
I=\int_{0}^{1} x^{3 / 2}\left(1-x^{3}\right)^{1 / 2} d x \cong 0.28
$$

In the opposite limit $(6 \mu+p) R_{n} / 3 \sigma \ll 1$ the energy spectrum of the void is determined by

$$
\begin{aligned}
& \Delta E_{n} \cong 2^{12 / 7} 3^{2 / 7} \pi \frac{\sigma^{5 / 7}}{\rho^{2 / 7}} \hbar^{4 / 7}\left(n+\frac{3}{4}\right)^{4 / 7}, \\
& R_{n}^{2} \cong \frac{\Delta E_{n}}{4 \pi \sigma} .
\end{aligned}
$$

A spectrum similar to Eq. (19) was obtained by Lifshitz and Kagan [10] for the energy of quantum nuclei and by Khrapak $[11,12]$ for the energy spectrum of microscopic bubbles (bublons) in ideal incompressible liquids.

From Eqs. (16) and (17) it follows that the creation energy and size of the voids are quantized and can not be arbitrary small. Ground state with minimal values of the $E_{n}$ and $R_{n}$ are realized for $n=0$. Owing to their quantum nature, the zero-oscillations of voids cannot decay, for example, as result of the acoustic radiation. This gives reason to think that within the continuum matter model, the ground state of voids corresponds to the vacancies in real crystals.

Below we estimate some properties of vacancies in solid Ar using the proposed model. At $T=0$ the density of solid $\mathrm{Ar}$ is $\rho_{s}=1.77 \mathrm{~g} / \mathrm{cm}^{3}$ and the shear modulus is $\mu=14.6$ kbar [13]. Assuming that at $T=0$ a contribution of the surface force can be neglected, then for $p=0$ the vacancy ground state energy and radius are given by

$$
\begin{aligned}
& \Delta E_{0}=\frac{3^{9 / 8} \pi}{2^{3 / 4} I^{3 / 4}} \frac{\mu^{5 / 8} \hbar^{3 / 4}}{\rho_{s}^{3 / 8}} \cong 1290 \mathrm{~K}, \\
& R_{0}=\left(\frac{3^{3} \hbar^{2}}{2^{10} I^{2} \mu \rho_{s}}\right)^{1 / 8} \cong 0.8 \cdot 10^{-8} \mathrm{~cm} .
\end{aligned}
$$

This value of $\Delta E_{0}$ is in qualitative agreement with theoretical and experimental estimations of the monovacancy formation energy $E_{v} \cong 905 \mathrm{~K}$ (see, for example, [1]). Equation (20) is valid when $2 \mu R_{0} / \sigma_{s} \gg 1$. To check the validity of this inequality one has to know the surface ten- 


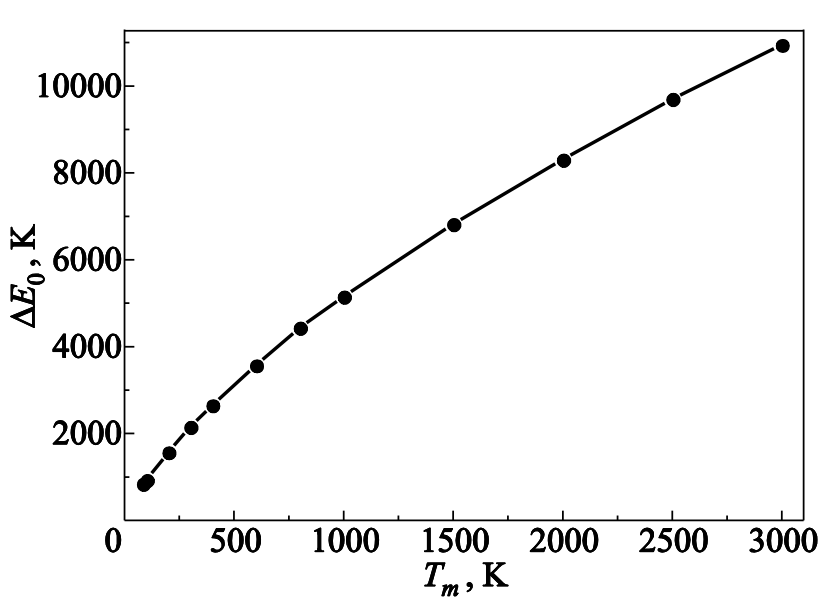

Fig. 1. The energy of the vacancy creation in solid Ar as a function of the melting temperature.

sion $\sigma_{s}$ of the solid Ar. It can be estimated by means of the parachore formula [14] according to which $\sigma \sim \rho^{4}$. Using well known surface tension of liquid Ar at the triple point $\sigma_{l}=13.4 \mathrm{dyn} / \mathrm{cm}$ and density $\rho_{l}=1.42 \mathrm{~g} / \mathrm{cm}^{3}$ we get

$$
\sigma_{s} \cong\left(\rho_{s} / \rho_{l}\right)^{4} \sigma_{l} \cong 32.4 \mathrm{dyn} / \mathrm{cm} \text {. }
$$

This gives $2 \mu R_{0} / \sigma_{s} \cong 11$ and thus the neglect of the surface energy is justified.

At the Ar triple point $T=T_{t}=83.8 \mathrm{~K}, \rho_{s}=1.62 \mathrm{~g} / \mathrm{cm}^{3}$, $\mu \cong 6.00 \mathrm{kbar}[13], \quad \sigma_{s} \cong 22.7 \mathrm{dyn} / \mathrm{cm}$. According to Eqs. (16) and (17) $\Delta E_{0} \cong 880 \mathrm{~K}$ (contribution to $\Delta E_{0}$ from the surface energy amounts to $\sim 15 \%$ ). The shear modulus $\mu=\rho_{s} c_{t}^{2}$ ( $c_{t}$ is the transverse sound velocity) increases with the melting temperature $T_{m}$ and density $\rho_{m}$. In $\operatorname{Ar} c_{t}$ was measured along its solidus in of relatively wide temperature region, from $T_{t}$ to $205.6 \mathrm{~K}[15,16]$. Corresponding values of $\mu$ were reported by Burakovsky et al. [13] together with an analytical formula for $\mu\left(T_{m}\right)$, which permits an extrapolation to extremely high temperatures $T_{m} \sim 3000 \mathrm{~K}$. In Fig. 1 the dependence of $\Delta E_{0}$ on $T_{m}$, following from Eqs. (16) and (17), with $\mu\left(T_{m}\right)$ from [13] and $\rho_{m}, p_{m}$ from [17] is shown. One can see that with a good accuracy $\Delta E_{0} / T_{t} \cong 10.5$ which is in reasonable agreement with the approximate relation $E_{v} / T_{t} \cong 9.4$ obtained by Bhatia and March [18] for monovacancies in solid Ar. The ratio $\Delta E_{0} / T_{m}$ decreases with the melting temperature. Direct comparison with experiments, where the constancy of the ratio $E_{v} / T_{m}$ is peculiar to classical rare gas crystals, a number of metals [19] and even for quantum crystals [20,21] is problematic for (at least) two reasons: First, the enthalpy of the vacancy formation is usually measured instead of $E_{v}$ and the contribution of the nonconfigurational entropy (which has to grow with $T_{m}$ ) is difficult to detach. Second, the shear modulus increases with the frequency of an external force (see, for example, [22]). The frequency of the void wall oscillations
$\omega_{0}=\Delta E_{0} / \hbar \sim 10^{14} \mathrm{~Hz}$ at the triple point, and it increases with $T_{m}$. This effect was not taken into account in calculating $\mu\left(T_{m}\right)$ [13]. Unfortunately, the dependence $\mu(\omega)$ at very high frequencies is unknown.

The local undamped oscillations of matter surrounding the vacancy are not artifacts of the continuum matter model. In real crystals the vacancy formation has to result in reconstruction of the oscillation spectrum and in appearing of a new quantized mode. This effect may be important for development the vacancy theory of melting. The existing theories interpret the melting as the first order phase transition in the vacancy subsystem: At the melting line the equilibrium between crystalline system with relatively low concentration of vacancies, $c=n_{v} / n_{a} \sim 10^{-3}$, and quasicrystalline system (liquid melt) with relatively high $c \sim 10^{-1}$ takes place. However, properties of vacancies in the melt are unknown. We suppose that owing to the high frequency of the vacancy wall oscillations, the shear modulus does not change significantly upon melting and vacancies in a liquid preserve main properties of vacancies in a solid.

\section{Acknowledgments}

The work was supported by the program of the Presidium of Russian Academy of Science "Matter at High Density Energy".

1. A.V. Chadwick and H.R. Glyde, Rare Cas Solids, M.L. Klein and J.A. Venables (eds.), Vol. 2, Academic Press, London (1977), p. 1151.

2. Y.I. Frenkel, Kinetic Theory of Liquids, Dover, New York (1955).

3. H. Eyring and T. Ree, Proc. Nat. Acad. Sci. 47, 526 (1965).

4. L.S. Metlov, Phys. Rev. Lett. 106, 165506 (2011).

5. J. Solka, A.J. Dyson, G. Steinebrunner, B. Kirchner, and H. Huber, Chem. Phys. 224, 253 (1997).

6. P.M. Agrawal, B.M. Rice, and D.L. Thompson, J. Chem. Phys. 118, 9680 (2003).

7. C.C. Matthai and N.H. March, Phys. Chem. Liq. 44, 329 (2006).

8. R.O. Simmons, Mat. Chem. Phys. 50, 124 (1997).

9. L.D. Landau, L.P. Pitaevskii, E.M. Lifshitz, and A.M. Kosevich, Theory of Elasticity, Elsevier Science, Oxford (1986).

10. I.M. Lifshitz and Yu. Kagan, Sov. Phys. JETP 35, 206 (1972).

11. A.G. Khrapak, JETP Lett. 47, 445 (1988).

12. A.G. Khrapak, JETP Lett. 51, 458 (1990).

13. L.C. Burakovsky, W. Greeff, and D.L. Preston, Phys. Rev. B 67, 094107 (2003).

14. J.O. Hirschfelder, C.F. Curtiss, and R.B. Bird, Molecular Theory Gases and Liquids, Wiley, New York (1954).

15. H.R. Moeller and C.F. Squire, Phys Rev. 151, 689 (1966).

16. K. Ishizaki, I.L. Spain, and P. Boisaitist, J. Chem. Phys. 63, 1401 (1975). 
17. C.-S. Zha, R. Boehler, D.A. Young, and M. Ross, J. Chem. Phys. 85, 1034 (1986).

18. A.B. Bhatia and N.H. March, J. Chem. Phys. 80, 2076 (1984).
19. J.M. Bernasconi, N.H. March, and M.P. Tosi, Phys. Chem. Liq. 16, 39 (1986).

20. R.O. Simmons, J. Phys. Chem. Solids 55, 895 (1994).

21. V. Tozzini and M.P. Tosi, Philos. Mag. Lett. 76, 377 (1997).

22. J. Beamish, J. Low Temp. Phys. 168, 194 (2012). 